\title{
O conhecimento de mulheres acerca do rastreamento do câncer de mama e suas implicações
}

\author{
The knowledge of women about breast cancer screening and its implications
}

\author{
Amanda Azevedo', Amanda Lúcia Ramos', Ana Caroline Vicenzi Gonçalves ${ }^{1}$, \\ Camila Fernandes de Souza ${ }^{1}$, Gabriela Silva Batista ${ }^{1}$, Roberta Bessa Veloso Silva ${ }^{2}$, \\ Edilaine Assunção Caetano de Loyola ${ }^{3}$
}

Azevedo A, Ramos AL, Gonçalves ACV, Souza CF, Souza CF, Batista GS, Silva RBV, Loyola-Caetano EA. O conhecimento de
mulheres acerca do rastreamento do câncer de mama e suas implicações / Women's knowledge about breast cancer screening and its implications. Rev Med (São Paulo). 98 maio-jun.;98(3):187-93.

RESUMO: Introdução: O câncer de mama é a principal causa de morte por câncer na população feminina e a segunda neoplasia mais frequente no mundo. A detecção precoce da doença, principalmente por meio da mamografia, é fundamental para reduzir o número de óbitos e melhorar o prognóstico. Objetivo: analisar o conhecimento de mulheres entre 35 e 69 anos cadastradas em Estratégias de Saúde da Família (ESF) de Alfenas - MG acerca do rastreamento do câncer de mama. Métodos: Estudo quantitativo, descritivo e transversal no qual foram aplicados questionários em uma população amostral, selecionada a partir do total de mulheres cadastradas em cinco ESF de Alfenas - MG, sendo entrevistadas 224 mulheres a partir de questionário estruturado. Os dados foram analisados utilizandose a estatística multivariada por meio da técnica de componentes principais. Todos os critérios éticos foram respeitados. Resultados: As ações preventivas de rastreamento influenciaram na redução da mortalidade e melhor prognóstico da doença. A terapia de reposição hormonal está correlacionada com as alterações encontradas nas mamas. A idade da primeira menstruação mostrou-se correlacionada às mulheres que já estão na menopausa, características essas consideradas de risco para o desenvolvimento da neoplasia de mama. A regularidade com que as mulheres realizam a mamografia depende de seu conhecimento acerca deste exame, considerando-se a realização e prática do autoexame importante. Verificou-se que o uso de anticoncepcional está diretamente relacionado com a idade e o estado civil, além de alterações encontradas no exame clínico das mamas. Estar na menopausa esteve diretamente relacionado à realização de mamografia. Fatores de risco para câncer de mama como tabagismo, dieta rica em gordura e etilismo, além de estarem altamente correlacionados, apresentaram uma relação inversa com a realização da mamografia. Conclusões: o conhecimento acerca dos exames preventivos e da sua importância está relacionado com a regularidade com que as mulheres os realizam.

Descritores: Neoplasias de mama/diagnóstico; Mamografia; Programas de rastreamento; Detecção precoce do câncer; Conhecimento; Mulheres.

\begin{abstract}
Introduction: Breast cancer is the leading cause of cancer death in the female population, and the second most frequent neoplasm in the world. The early detection of neoplasms, mainly through mammography, is fundamental to reduce the number of deaths and improve the prognosis. Objective: analyze the knowledge of women between 35 and 69 years of age enrolled in Family Health Strategies (ESFs) of Alfenas - MG, about the screening of breast cancer. Methods: A quantitative, descriptive and cross - sectional study in which questionnaires were applied in a sample population, selected from the total number of women enrolled in five ESFs in Alfenas - MG, and 224 women were interviewed from a structured questionnaire. Data were analyzed using multivariate statistics using the principal components technique. All ethical criteria have been respected. Results: The preventive actions of screening influenced the reduction of mortality and better prognosis of the disease. Hormone replacement therapy is correlated with changes found in breasts. The age of the first menstrual period was shown to be correlated to the women who are already in menopause, characteristics considered to be at risk for the development of breast cancer. The regularity with which women perform mammography depends on their knowledge about this examination, considering the performance and practice of the important self-examination. It was verified that the use of contraceptives is directly related to age and marital status, as well as alterations found in the clinical examination of the breasts. Being in menopause was directly related to mammography. Risk factors for breast cancer, such as smoking, high fat diet and alcoholism, besides being highly correlated, presented an inverse relationship with mammography. Conclusions: knowledge about preventive exams and their importance is related to the regularity with which women perform them.
\end{abstract}

Keywords: Breast neoplasms/diagnosis; Mammography; Mass screening; Early detection of cancer; Knowledge; Women.

Apresentado: XIV e XV Seminários de Iniciação Científica da UNIFENAS - Alfenas, MG, 23 out. 2015 ; 21 out. 2017.

Agência de Fomento: Fundação de Amparo à Pesquisa de Minas Gerais/ FAPEMIG - Programa de Bolsas de Iniciação Científica.

1. Acadêmica do curso de Medicina da Universidade José do Rosário Vellano - UNIFENAS, Alfenas-MG. ORCID: Azevedo A - https://orcid.org/00000002-5819-2741; Ramos AL - https://orcid.org/0000-0002-3557-3576; Gonçalves ACV - https://orcid.org/0000-0002-6421-8987; Souza CF - https:// orcid.org/0000-0002-1786-2585; Batista GS - https://orcid.org/0000-0003-39880343. Email: a.azevedocv@gmail.com; amandaramos500@hotmail. com; anacarolinevicenzi@gmail.com; camilafernandes0100@gmail.com; med.gabrielasb@gmail.com.

2. Doutora em Estatística e Experimentação Agropecuária/UFLA. Docente na Universidade José do Rosário Vellano - UNIFENAS. https://orcid. org/0000-0003-4794-5872. Email: bessaveloso@yahoo.com.br.

3. Doutora e Mestra em Ciências pela Escola de Enfermagem de Ribeirão Preto da Universidade de São Paulo (EERP/USP). Especialização em Cuidados Pré-Natais pela Universidade Federal de São Paulo e Especialização em Formação em Educação Profissional Técnica na área da Saúde pela Fundação Oswaldo Cruz/Escola Nacional de Saúde Pública. Docente na Universidade José do Rosário Vellano - UNIFENAS. https://orcid.org/0000-0002-44411229. Email: dipatinga@hotmail.com.

Endereço para correspondência: Amanda Azevedo. Rua 13 de Maio, nº 371, Centro. Cabo Verde, MG, BR. CEP: 37880-000. E-mail: a.azevedocv@ gmail.com. 


\section{INTRODUÇÃO}

$\mathrm{O}$ câncer de mama é a segunda neoplasia mais frequente no mundo, sendo a mais comum entre as mulheres. Na população feminina, o carcinoma mamário tem ocupado a primeira posição no que se refere à causa de morte por câncer ${ }^{1}$. Segundo os dados do Instituto Nacional de Câncer José de Alencar Gomes da Silva INCA, são estimados 59.700 casos para o ano de 2018. Sua incidência vem crescendo, sobretudo, após os $50 \mathrm{anos}^{2}$. Em virtude dessa alta taxa de novos casos e mortalidade, têm-se buscado ações que visam o rastreamento da doença, a fim de se detectar precocemente a neoplasia ${ }^{3}$.

A realização do rastreamento mamográfico é considerada, atualmente, a forma mais eficiente para a detecção precoce da neoplasia mamária ${ }^{4}$. O rastreamento é a aplicação sistemática de teste em população presumivelmente assintomática, que se destina a identificar indivíduos com uma anormalidade sugestiva de câncer ${ }^{5}$ Assim, a mamografia é considerada o método padrão-ouro preconizado para o rastreamento no Brasil, sendo que a recomendação para as mulheres de 50 a 69 anos é a realização deste exame a cada dois anos ${ }^{4}$. A mamografia, nesta faixa etária, e a periodicidade bienal é a rotina adotada na maioria dos países que implantaram o rastreamento organizado do câncer de mama. Esta rotina se baseia na evidência científica do benefício desta estratégia na redução da mortalidade neste grupo ${ }^{6}$.

As novas Diretrizes para a detecção precoce do câncer de mama no Brasil trouxeram a novidade de ausência de recomendação do Exame Clínico das Mamas (ECM) para a detecção precoce do câncer de mama para as mulheres não pertencentes ao grupo de risco elevado. Baseadas em evidências científicas, as diretrizes enfatizam que o balanço entre possíveis danos e benefícios é incerto, mesmo o exame sendo executado por profissional treinado; porém o ECM se constitui em parte do exame físico da mulher e deve continuar fazendo parte da rotina de cuidados nas consultas ${ }^{4,5}$.

Com relação ao Autoexame de Mamas (AEM), apesar de ter deixado de ser recomendado nacionalmente para todos os grupos de mulheres em idade de rastreamento desde o consenso de 2004 ${ }^{7}$, ainda é comumente encontrado em materiais educativos distribuídos no país. O estudo de Moraes et $\mathrm{al}^{5}$. indica que essa prática ainda é muito difundida, uma vez que $70 \%$ dos profissionais entrevistados orientam as usuárias sobre técnicas para o autoexame das mamas, devendo, portanto, ser pesquisada.

Quanto às mulheres com risco elevado, o Ministério da Saúde inclui aquelas com história familiar de câncer de mama em parente de primeiro grau antes dos 50 anos ou de câncer bilateral ou de ovário em qualquer idade; história familiar de câncer de mama masculino; e diagnóstico histopatológico de lesão mamária proliferativa com atipia ou neoplasia lobular in situ? .
A definição sobre a forma de rastreamento da mulher de alto risco não tem ainda suporte nas evidências científicas atuais e é variada a abordagem deste grupo nos programas nacionais de rastreamento. Recomenda-se que as mulheres com risco elevado de câncer de mama tenham acompanhamento clínico individualizado ${ }^{8}$.

Deste modo, é importante analisar o conhecimento das mulheres acerca do câncer de mama. A pesquisa realizada por Migowski et al. ${ }^{9}$ acerca dos desafios da implantação das novas diretrizes para o controle do câncer de mama no país apontou que, hoje, prevalecem no país práticas de rastreamento não baseadas nas melhores evidências disponíveis e que trazem, provavelmente, mais danos do que benefícios para a população,. Soma-se a isso que quesitos centrais como controle da qualidade do rastreamento, adesão às recomendações de rastreamento, distribuição adequada de mamógrafos, qualidade das mamografias e priorização de estratégias para casos sintomáticos ainda permanecem como questões de difícil resolução.

A ultrassonografia tem demostrado importante papel sendo suporte no diagnóstico, além de oferecer dados que são utilizados para estadiar a doença e escolher o melhor tratamento ${ }^{10}$. Este exame é o escolhido para mulheres com menos de 35 anos, mas também pode ser indicado no caso de mamas densas, nódulos palpáveis com ou sem mamografia negativa, processos inflamatórios e mulheres grávidas que apresentem sintomas mamários.

Assim, o câncer como uma enfermidade crônica é caracterizado pelo crescimento celular desordenado, o qual é resultante de alterações no código genético ${ }^{11}$. Quanto aos aspectos genéticos do câncer humano, há uma predisposição familiar e também fatores ambientais predisponentes ao câncer, como as radiações, alguns vírus e substâncias químicas consideradas carcinogênicas ${ }^{11}$. As atuais mudanças de estilo de vida constituem fatores de risco para a ocorrência da doença. Dentre essas mudanças, podem-se destacar a ausência da maternidade ou início dela após os 30 anos, intervenção hormonal, sedentarismo, má alimentação, obesidade, tabagismo, consumo excessivo de álcool e história familiar, sendo a idade o mais importante fator de risco para a detecção da neoplasia de mama ${ }^{12}$.

Estudos $^{12-15}$ observacionais indicam que $o$ comportamento humano relacionado ao estilo de vida, o que inclui modificações na dieta e na prática de atividade física, pode contribuir para o aumento da incidência do câncer de mama. As neoplasias também são influenciadas pelas condições ambientais, não sendo determinadas apenas por fatores genéticos ${ }^{14}$. As populações que migram do país de origem para outras localidades apresentaram mudanças nas taxas de incidência de câncer ${ }^{11}$.

Uma vez detectada a neoplasia mamária, destacamse como as principais terapias utilizadas para o tratamento locorregional do carcinoma: a cirurgia e a radioterapia. Com ação sistêmica, está disponível a quimioterapia, a 
hormonioterapia e a imunoterapia ${ }^{12}$.

No entanto, quando o diagnóstico da doença é feito em estágios avançados, as chances de cura são reduzidas. Este fator também constitui uma das causas responsáveis pelas altas taxas de mortalidade da doença ${ }^{13}$. As condições socioeconômicas, o confinamento geográfico e as distintas modalidades de acesso individual aos serviços de saúde levam ao aparecimento de barreiras prejudiciais à descoberta precoce das neoplasias ${ }^{16}$.

$\mathrm{O}$ rastreamento do câncer de mama possibilita $\mathrm{O}$ diagnóstico da doença em fase inicial, permitindo que aumente a possibilidade de cura e que sejam utilizadas formas de tratamentos menos radicais e sistêmicas e, portanto, diminuem suas consequências, conduzindo a uma recuperação mais efetiva ${ }^{8}$.

O rastreamento pode ser oportunístico ou organizado. No primeiro, o exame de rastreio é ofertado às mulheres que oportunamente chegam às unidades de saúde, enquanto o modelo organizado é dirigido às mulheres elegíveis de uma dada população que são formalmente convidadas para os exames periódicos. A experiência internacional tem demonstrado que esse modelo apresenta melhores resultados e menores custos ${ }^{6}$.

No Brasil, o programa para o rastreamento do câncer mamário tem caráter oportunístico, e cada localidade vem desenvolvendo ações isoladas no que diz respeito ao controle do câncer de mama; portanto, é de extrema importância avaliar o conhecimento de mulheres acerca do câncer de mama e suas implicações na detecção das neoplasias mamárias ${ }^{5,10,16}$.

Assim, quanto ao conhecimento das mulheres frente a essas neoplasias, um estudo realizado em uma Estratégia Saúde da Família - ESF, em Campina Grande, na Paraíba, revelou que 94,59\% das entrevistadas tem conhecimento do câncer de mama, 83,7\% relataram saber como se prevenir, 67\% afirmaram realizar o AEM, das quais, apenas $16 \%$ o fazem regularmente. Além disso, esse estudo mostrou que grande parte do sexo feminino ainda apresenta dúvidas a respeito do assunto, indicando a importância de ampliar o conhecimento sobre como fazer uma prevenção da doença de forma eficaz ${ }^{17,18}$.

Diante do exposto, este trabalho teve como objetivo analisar o conhecimento de mulheres entre 3569 anos cadastradas na Estratégia de Saúde da Família (ESF) de Alfenas - MG, acerca do rastreamento do câncer de mama.

\section{MÉTODO}

Para medir o conhecimento da população investigada foi conduzido um estudo quantitativo, descritivo e transversal. A cidade de Alfenas, localizada no sul do estado de Minas Gerais, com população de aproximadamente 79.000 habitantes, em 2018, conta com treze ESF em funcionamento, conferindo uma cobertura de aproximadamente $70 \%$ de ESF, das quais foram selecionadas cinco, a saber: Jardim Primavera, Recreio Vale do Sol, São Carlos, Unisaúde, Vila Betânia. A escolha dessas unidades se deu por serem vinculadas à Universidade José do Rosário Vellano/UNIFENAS, campus de Alfenas, MG, para atividades práticas e campo de estágio.

Umaamostraaleatóriafoicalculada estatisticamente após realização da contagem da população alvo nas fichas de cadastramento de mulheres residentes nas áreas adstritas das ESF. A amostra em estudo foi constituída por mulheres na faixa etária estipulada pelo Sistema Único de Saúde (SUS) para o início das políticas de rastreamento do câncer de mama, a partir do total de mulheres que estavam cadastradas no e-SUS Atenção Básica. Deste modo, considerou-se a idade mínima sugerida, 35 anos, para o início de atenção às mulheres que apresentarem risco elevado para o desenvolvimento da neoplasia e a idade máxima estipulada para realização de mamografia, 69 anos.

Assim, totalizavam 3128 mulheres cadastradas nos registros das ESF, na faixa etária selecionada. A partir deste total, foi realizado o cálculo amostral perfazendo 434 mulheres. A coleta dos dados foi realizada a partir de busca das mulheres em seus domicílios nas áreas de abrangência das cinco unidades de saúde selecionadas para o estudo. Foram realizadas três tentativas em horários diferentes no intuito de minimizar ao máximo o número de perdas.

Desse modo, após todas as tentativas, foram avaliadas 224 mulheres que se encontravam em conformidade com os critérios pré-estabelecidos para o rastreamento do câncer de mama. Portanto, as mulheres cadastradas na ESF da cidade de Alfenas-MG foram convidadas a participarem deste estudo para a análise de seus conhecimentos acerca do rastreamento do câncer de mama e a sua relevância para o bom prognóstico da doença, após serem identificadas a partir do E-SUS.

Em seguida, foi aplicado um questionário semiestruturado composto por 31 questões e seus subitens referentes aos dados de identificação, como idade, estado civil, raça autodeclarada, ocupação e plano de saúde; dados sobre conhecimento sobre o câncer de mama e sobre os fatores de risco para desenvolvimento da doença, a saber: histórico de câncer de mama na família, regularidade de comparecimento à consulta ginecológica, se a mulher sabe o que é câncer de mama e se é ou já foi portadora, se já realizou ou realiza alguma terapia de reposição hormonal, se possui filhos e se os amamentou, idade da primeira gestação, idade da menarca e se a mulher está na menopausa e, caso positivo, com qual idade entrou na menopausa (antes ou após os 55 anos); se faz uso de anticoncepcional, bebida alcóolica, incluindo a frequência, fumo, incluindo a quantidade por dia, e se considera sua dieta rica em gorduras.

Quanto ao ECM, foi indagado se a mulher considera 
o ECM importante; se, pelo menos uma vez por ano, as suas mamas teriam sido examinadas por médico ou enfermeiro da ESF e em qual momento foi realizada esta avaliação: durante o exame ginecológico preventivo (Papanicolaou), durante a consulta de enfermagem, na consulta médica, na visita domiciliar ou em outro momento, especificando qual e se foi encontrada alguma alteração ao examinar as mamas.

Sobre a mamografia, foi questionado se a mulher sabia o que era este exame e, caso positivo, se ela tinha conhecimento sobre a importância do mesmo na prevenção e diagnóstico do câncer de mama. Também foi perguntado à entrevistada se ela sabia com qual idade deve ser feita a primeira mamografia e se ela já havia feito alguma vez e com qual regularidade.

A respeito do AEM, as perguntas realizadas foram: se a mulher achava importante realizar tal exame, se sabia fazê-lo, se o realizava mensalmente, em qual época do período menstrual e com quem teria aprendido a praticar.

Finalmente, era questionado à mulher se alguma vez ela havia feito ultrassom de mamas e com qual frequência e se ela confiava em exames de imagem. Também se, para a mulher, havia benefícios na associação do AEM, com o ECM, mamografia e ultrassonografia de mamas no diagnóstico do carcinoma mamário.

Tal instrumento foi elaborado pelas autoras e validado em forma e conteúdo por peritos, docentes da área de saúde da mulher; e, a coleta de dados somente foi iniciada após aprovação deste trabalho pelo Comitê de Ética em Pesquisa Humana - CEP - da Universidade José do Rosário Vellano/UNIFENAS, sob o parecer $n^{\circ}$ 909.301. Todas as participantes assinaram o Termo de Consentimento Livre e Esclarecido (TCLE).

Foi construído um banco de dados no Microsoft Office Excel 2010 e realizada dupla digitação dos dados para validação. Em seguida, os dados foram analisados utilizando-se a estatística multivariada por meio da técnica de componentes principais, que são combinações lineares de $p$ variáveis correlacionadas.

A estatística multivariada tem como ingrediente básico a observação de diversas variáveis simultaneamente nas unidades amostrais ou experimentais. A análise, a descrição e a inferência são realizadas com base nas respostas simultâneas valendo-se da estrutura de correlação entre as variáveis ${ }^{19}$.

As técnicas multivariadas têm sido regularmente aplicadas em várias investigações científicas nas áreas de biologia, física, sociologia e ciências médicas. Nos estudos em que as reações de pacientes a um determinado tratamento são mensuradas em algumas variáveis e, possuem difícil diagnóstico, as técnicas multivariadas podem ser usadas para construir uma medida de resposta simples ao tratamento, na qual é preservada a maior parte da informação da amostra e das múltiplas variáveis respostas $^{19}$.

Neste trabalho, as questões foram codificadas de acordo com o número de alternativas, exceto a Questão 1, referente à idade. Às questões com respostas dicotômicas foram atribuídos 0 e 1 . Para as questões com mais de duas alternativas, foram atribuídos os escores $i=1,2,3,4,5$, dependendo do número de alternativas. Sendo assim, foi possível avaliar o grau de correlação entre as variáveis simultaneamente.

A análise estatística foi realizada no software R (CORE TEAM, 2018) ${ }^{20}$.

\section{RESULTADOS}

No mapa de variáveis, Figura 1, pode-se observar, no primeiro quadrante superior da direita, que as variáveis (Questões), denominadas de Q14, Q15_1, Q16, Q20, Q21, Q23, Q24, Q29 e Q30 são altamente correlacionadas e diretamente proporcionais, pois têm a mesma direção. Estas questões referem-se à terapia de reposição hormonal (Q14); idade em que entrou na menopausa (Q15_1); uso de anticoncepcional (Q16); realização de exame das mamas pelo médico ou enfermeiro uma vez por ano (Q20); importância do exame clínico (Q21); alteração nas mamas (Q23); conhecimento sobre mamografia (Q24); importância do autoexame (Q29), conhecimento sobre realizar o autoexame $(\mathrm{Q} 30)$.

No segundo quadrante inferior da direita, na Figura 1 , verifica-se que as variáveis supramencionadas também estão correlacionadas com a idade (Q1), estado civil (Q2), idade que teve a primeira menstruação (Q13), estar na menopausa (Q15), número de cigarros consumidos por dia (Q18_1), sendo estas altamente correlacionadas entre si e diretamente proporcionais.

Em se tratando das variáveis que estão no terceiro quadrante inferior da esquerda, Figura 1, pode-se observar alta correlação entre a raça $(\mathrm{Q} 3)$, regularidade que realiza a mamografia (Q28), momento em que o profissional examinou as mamas (Q22), como aprendeu a realizar o autoexame (Q30_1), período menstrual em que realiza o autoexame (Q31_2), frequência de uso de anticoncepcional (Q17_1), sendo diretamente proporcionais.

Na Figura 1, ao comparar o primeiro quadrante (superior direito) com o terceiro, inferior esquerdo, verifica-se que a idade (no momento da realização da entrevista); a idade em que entrou na menopausa; o conhecimento sobre a importância do exame clínico; sobre alteração nas mamas; sobre a mamografia; e importância do autoexame e o conhecimento em como realizar o autoexame estão correlacionadas com a raça. Já a regularidade com que realiza a mamografia; momento em que o profissional examinou as mamas; como aprendeu a realizar o autoexame; período menstrual em que realiza o autoexame e a frequência de uso de anticoncepcional, porém são inversamente proporcionais.

No quarto quadrante superior da esquerda, na Figura 1, observa-se alta correlação entre a frequência de 
uso de anticoncepcional (Q17); hábito de fumar (Q18); dieta rica em gorduras (Q19); importância do autoexame na prevenção do câncer de mama (Q24_1); se já realizou mamografia $(\mathrm{Q} 25)$ e a frequência com que realiza o autoexame (Q31_1). Estas variáveis estão correlacionadas com a idade (Q1); estado civil (Q2); idade em que teve a primeira menstruação (Q13); encontra-se na menopausa (Q15); número de cigarros consumidos por dia (Q18_1).
O mapa de observações (Figura 2) mostra a distribuição das participantes ao longo dos quatro quadrantes. Observa-se uma maior concentração no primeiro (superior à direita), segundo (inferior à direita) e quarto (superior à esquerda), indicando uma correlação entre as características das participantes com as variáveis localizadas nos mesmos quadrantes, no mapa de variáveis (Figura 1).



Figura 1. Mapa de variáveis evidenciando a correlação entre as variáveis

Individuals factor map (PCA)



Figura 2. Mapa de observações com a distribuição dos participantes nos quatro quadrantes do mapa 


\section{DISCUSSÃO}

A realização de ações preventivas que visam o rastreamento e diagnóstico precoce do câncer de mama influencia diretamente na redução da mortalidade e em um melhor prognóstico da doença. Este resultado está de acordo com os estudos que demonstraram que a mamografia constitui o principal método para detecção precoce do câncer de mama' ${ }^{1,910}$.

Pode-se observar que a terapia de reposição hormonal está correlacionada com as alterações encontradas nas mamas. Entretanto, os achados na literatura mostraram que esta associação ainda é incerta. Verificou-se que a administração de estrógeno isolado ou junto à progesterona elevou a porcentagem de densidade mamária, sendo este um fator de risco para o desenvolvimento da neoplasia. Contudo, não há dados suficientes para avaliar as diferenças de casos novos da neoplasia mamária usando diferentes tipos e vias de administração de estrogênio ${ }^{21}$.

A idade da primeira menstruação mostrou-se correlacionada às mulheres que já estão na menopausa. Estas características reprodutivas são consideradas de risco para o desenvolvimento da neoplasia, uma vez que a doença é dependente de estrogênio. Este fato justifica as condições como menarca precoce (11 anos ou menos) e a menopausa tardia (55 anos ou mais) constituírem-se fatores de risco para o desenvolvimento do câncer de mama $^{12}$.

Observou-sequearegularidade comqueas mulheres realizam a mamografia depende de seu conhecimento acerca deste exame, considerando-se a realização e prática do AEM importante. Este conhecimento sobre o câncer de mama e a detecção precoce fomenta a sua motivação com a saúde, fazendo com que isto as influencie na realização de exames que possibilitem a identificação da neoplasia. Nos dias atuais, o AEM não se constitui mais em uma estratégia isolada para a detecção precoce do câncer de mama, entretanto, tem-se mostrado eficaz como forma de ação para conhecimento do próprio corpo ${ }^{10}$.

Ao final da década de 1990, ensaios clínicos mostraram que o AEM não reduzia a mortalidade por câncer de mama, uma vez que, quando um tumor é detectado pela própria mulher, já se encontra em grande tamanho. Desse modo, diversos países passaram a usar a estratégia breast awareness, que quer dizer "estar alerta para a saúde das mamas" e foca em orientar as mulheres a conhecerem o próprio corpo e a estarem atentas para eventuais mudanças corporais atípicas. A orientação é que a mulher realize a autopalpação das mamas sempre que se sentir confortável para tal (seja no banho, no momento da troca de roupa ou em outra situação do cotidiano), sem nenhuma recomendação de técnica específica ${ }^{4,7}$.

Quanto ao uso de algum anticoncepcional, verificou-se que está diretamente relacionado com a idade e o estado civil. Além disso, foi possível detectar a dependência entre as alterações encontradas no ECM com a frequência de uso de anticoncepcional. Estes resultados são concordantes com os obtidos a partir de um estudo realizado no Reino Unido e que apontou que o uso de anticoncepcionais aumenta 1,24 vezes o risco de desenvolver câncer de mama ${ }^{5}$.

Outra observação importante foi em relação às mulheres que estão na menopausa já terem realizado mamografia. Resultados semelhantes apontaram que a maior busca pela prevenção, entre outros fatores, está associada ao tratamento para a menopausa. Portanto, a idade mostrou-se um fator determinante e contribuinte na prevenção da neoplasia ${ }^{13}$.

Fatores de risco para câncer de mama como tabagismo, dieta rica em gordura e etilismo, além de estarem altamente correlacionados, apresentaram uma relação inversa com a realização da mamografia e do ECM quando são realizados pelo profissional de saúde, indicando a necessidade de se intensificar o rastreio nesta população com fatores de risco modificáveis para a doença ${ }^{12}$.

\section{CONCLUSÃo}

Torna-se evidente a importância do rastreamento do câncer de mama, uma vez que possibilita um diagnóstico precoce e, posteriormente, o tratamento da doença. A implementação de programas de rastreamento constitui a principal estratégia capaz de reduzir a mortalidade por câncer de mama, sendo imprescindível o conhecimento da população acerca das formas de se detectar essa neoplasia. É necessário que as mulheres busquem o SUS, para que sejam oferecidos os serviços de atenção básica capazes de atuar na prevenção do desenvolvimento do câncer de mama.

Participação dos autores: Ramos AL, Gonçalves ACV, Souza CF, Batista GS - processo de coleta e análise dos dados e a elaboração do manuscrito. Silva RBV - Análises estatísticas e confecção do manuscrito. Azevedo A - Planejamento do projeto, processo de coleta e análise dos dados e a elaboração e revisão do manuscrito. Loyola-Caetano EA - planejamento do projeto, orientação científica, confecção do manuscrito e revisão.

\section{REFERÊNCIAS}

1. Medeiros GC, Bergmann A, Aguiar SS, Thuler LCS. Análise dos determinantes que influenciam o tempo para o início do tratamento de mulheres com câncer de mama no Brasil. Cad Saúde Pública. 2015;31(6):1269-82. doi: 10.1590/0102$311 \mathrm{X} 00048514$.
2. Brasil. Instituto Nacional de Câncer José de Alencar Gomes da Silva - INCA. Tipos de câncer. Câncer de mama. Disponível em: http://www2.inca.gov.br/wps/wcm/connect/ tiposdecancer/site/home+/mama/cancer_mama. 
3. Tomazelli JG, Silva GA. Rastreamento do câncer de mama no Brasil: uma avaliação da oferta e utilização da rede assistencial do Sistema Único de Saúde no período 20102012. Epidemiol Serv Saude. 2017;26(4):713-24. doi: $10.5123 / \mathrm{s} 1679-49742017000400004$

4. Brasil. Ministério da Saúde. Instituto Nacional de Câncer José Alencar Gomes da Silva. Diretrizes para a detecção precoce do câncer de mama no Brasil. Rio de Janeiro: INCA; 2015. Disponível em: https://www.inca.gov.br/sites/ufu. sti.inca.local/files//media/document//diretrizes_deteccao_ precoce_cancer_mama brasil.pdf.

5. Moraes DC, Almeida AM, Figueiredo EN, Loyola EAC, Panobianco MS. Opportunistic screening actions for breast cancer performed by nurses working in primary health care. Rev Esc Enferm USP. 2016;50(1):14-21. doi: 10.1590/ S0080-623420160000100002.

6. Brasil. Instituto Nacional de Câncer José de Alencar Gomes da Silva - INCA. Encontro Internacional sobre Rastreamento do Câncer de Mama. Rev Bras Cancerologia. 2009;55(2):97113. Disponível em: http://www1.inca.gov.br/rbc/n_55/v02/ pdf/03_evento_encontro_inter.pdf.

7. Brasil. Instituto Nacional de Câncer José de Alencar Gomes da Silva - INCA. Consenso de controle do dâncer de mama. Disponível em: http://www.inca.gov.br/publicacoes/ Consensointegra.pdf.

8. Souza NHA, Falcão LMN, Nour GFA, Brito JA, Castro MM, Oliveira MS. Câncer de mama em mulheres jovens: estudo epidemiológico no Nordeste brasileiro. SANARE. 2017;16(2):60-7. Disponível em: https://sanare.emnuvens. com.br/sanare/article/view/1179/640.

9. Migowski A, Dias MBK, Nadanovsky P, Azevedo e Silva G, Sant'Ana DR, Stein AT. Diretrizes para detecção precoce do câncer de mama no Brasil. III - Desafios à implementação. Cad Saude Publica. 2018;34(6):e00046317. doi: 10.1590/0102$311 \times 00046317$.

10. Gonçalves CV, Camargo VP, Cagol JM, Miranda B, Medonça-Sassi RA. O conhecimento de mulheres sobre os métodos para prevenção secundária do câncer de mama. Cien Saude Coletiva. 2017;22(12):4073-81. doi: 10.1590/1413812320172212.09372016 .

11. Inumaru LE, Silveira EA, Naves MMV. Fatores de risco e de proteção para câncer de mama: uma revisão sistemática. Cad Saude Publica. 2011;27(7):1259-70. doi: 10.1590/S0102-
311X2011000700002.

12. Rodrigues JD, Cruz MS, Paixão AN. Uma análise da prevenção do câncer de mama no Brasil. Cien Saude Coletiva. 2015;20(10):3163-76. doi 10.1590/1413812320152010.20822014 .

13. Silva PA, Riul SS. Câncer de mama: fatores de risco e detecção precoce. Rev Bras Enferm. 2011;64(6):1016-21. doi: 10.1590/ S0034-71672011000600005.

14. Tiezzi DG. Epidemiologia do câncer de mama. Rev Bras Ginecol Obstet. 2009;31(5):213-5. doi: 10.1590/S010072032009000500001 .

15. Tiezzi, DG. Rastreamento do câncer de mama no Brasil: ainda há tempo para refletirmos. Rev Bras Ginecol Obstet. 2013;35(9):385-7. doi: 10.1590/S0100-72032013000900001.

16. Schneider IJC, Giehl MWC, Boing AF, D'Orsi. Rastreamento mamográfico do câncer de mama no Sul do Brasil e fatores associados: estudo de base populacional. Cad Saude Publica. 2014;30(9):1987-97. doi: 10.1590/0102-311X00162313.

17. Araújo VS, Dias MD, Barreto CMC, Ribeiro AR, Costa AP, Bustorff LACV. Conhecimento das mulheres sobre o autoexame de mama na atenção básica. Rev Enf Ref. 2010;3(2):27-34. Disponível em: http://www.scielo. mec.pt/scielo.php?script $=$ sci_arttext $\&$ pid $=$ S 0874 $02832010000400003 \& \operatorname{lng}=$ pt.

18. Instituto Nacional do Câncer José de Alencar Gomes da Silva - INCA. Programa de Controle do Câncer de Mama. Disponível em: http://www2.inca.gov.br/wps/wcm/connect/ acoes_programas/site/home/nobrasil/programa_controle_ cancer_mama/deteccao_precoce.

19. Ferreira DF. Estatística multivariada. 3a ed. rev. ampl. Lavras: Ed. UFLA; 2018.

20. R Development Core Team. R: a language and environment for statistical computing. R Foundation for Statistical Computing, Vienna, Austria; 2018. Available from: https:// www.gbif.org/tool/81287/r-a-language-and-environment-forstatistical-computing.

21. Pardini D. Terapia de reposição hormonal na menopausa. Arq Bras Endocrinol Metab. 2014;58(2):172-81. doi: 10.1590/0004-2730000003044.

Recebido: 21.01 .19

Aceito: 